\title{
Distributed Power Control for Time Varying Systems: Performance and Convergence Analysis
}

\author{
Huanshui Zhang, Member, IEEE, Chung Shue Chen, Student Member, IEEE, and Wing Shing Wong, Fellow, IEEE
}

\begin{abstract}
This paper deals with a class of power control problems where the system link gains are assumed to be time varying and the signal-to-interference ratio (SIR) estimates are allowed to be corrupted with bounded noises. A simple control algorithm is devised by applying a distributed, fixed step approach. It is a feedback algorithm that requires only local information. By modifying the distributed, fixed step power control algorithm proposed by Sung and Wong, we obtain here a more robust version that can handle time varying link gains and measurement noises. The convergence property of the new algorithm is established and simulation studies were carried out to show that it is effective.
\end{abstract}

Index Terms-Cellular mobile system, distributed algorithm, power control, time-varying channels.

\section{INTRODUCTION}

$\mathbf{P}$ OWER control is an important component of resource management in a cellular communication system. The aim is to assign to each user a transmitter power level so that a global quality-of-service (QoS) performance index of the system is optimized. Various classes of power control problems can be formulated depending on the optimization objective. In the powerbalancing formulation, the problem has been identified as an eigenvalue problem for nonnegative matrices. In early works, such as [1]-[3], the proposed algorithms are synchronous, centralized, and link gains are assumed to be known. Due to practical considerations, distributed algorithms have been developed, see for example [4]-[7]. However, in these algorithms, either a global normalization factor or some communication among users are needed, which weakens the distributed nature of these algorithms.

Another approach for power control is QoS tracking, which is adopted by Foschini and Miljanic [8]. Instead of optimizing the worst case signal quality, the aim is to find a solution such that the power consumption is minimized while meeting QoS constraints. This approach has been further investigated in papers such as [9]-[11]. A distributed fixed step power control algorithm has been proposed by Sung and Wong [12], which

Manuscript received July 15, 2003; revised June 14, 2004, October 19, 2004. The work described in this paper was partially supported by a grant from the Research Grants Council of the HKSAR (Project no. CUHK4222/00E). The review of this paper was coordinated by Prof. C.-J. Chang. The first author is also supported by the National Natural Science Foundation of China (Project no. 60174017). This paper was presented in part at ICC'05.

H. Zhang is with Information and Control Research Center, Shenzhen Graduate School of Harbin Institute of Technology, HIT Campus, Shenzhen University Town, Xili, Shenzhen, P.R. China (e-mail: h_s_zhang@ hit.edu.cn).

C. S. Chen and W. S. Wong are with the Department of Information Engineering, Information and Systems Laboratory, Department of Information Engineering, The Chinese University of Hong Kong, Shatin, N.T., Hong Kong (e-mail: cschen9@ie.cuhk.edu.hk; wswong@ie.cuhk.edu.hk).

Digital Object Identifier 10.1109/TVT.2005.853470 effectively is a discretized version of the Foschini and Miljanic algorithm. A discrete algorithm is more applicable in practice since transmitter power outputs are typically quantized into discrete levels.

Another practical issue that needs to be addressed is measurement error. For example, it is shown in [13] that the Foschini and Miljanic algorithm does not converge to the optimal solution if measurements for signal-to-interference ratio (SIR) are corrupted by errors. The effect of measurement error in mobile radio environment has been studied in [14], and a distributed algorithm which is less sensitive to measurement error was proposed. However, it is only for time invariant systems. In [15], Ulukus and Yates applied the idea of stochastic approximation to a power control problem, thus providing a practical approach to the issue of measurement errors.

A third practical issue is the time varying nature of channels due to fading and user mobility. For most of the results reported in the literature, the underlying models tend to focus on cases where the link gains are assumed to be fixed or quasistationary. Some investigations on time varying systems can be found in [15]-[18]. For example, [16] gives a variant of the distributed constrained power control algorithm [19] by scaling up the SIR target with a close to optimal factor computed in advance from environment parameters and fading statistics with respect to an expected outage probability. In [13], the stochastic approximation technique is applied to derive a power update algorithm that can handle both measurement errors as well as randomness in link gains.

In this paper, we concentrate on studying a power control problem in the presence of fading as well as SIR measurement errors. The primary goal is to ensure that all users under time-varying channel conditions can maintain SNR above a predefined target and achieve a minimum performance threshold. A new, distributed, fixed step algorithm is obtained by building on the approach of [12]. The proposed algorithm extends the Foschini and Miljanic model to allow fading and measurement errors. It is established that under the algorithm, the SIR of each user converges to a target region. The size of the region depends, among other things, on the variances of the link gains and the measurement errors. The modified algorithm can operate under time varying channels and is robust to measurement errors. Convergence of the algorithm is proved as long as a feasible solution exists for an associated power control problem defined by the system bounds. The approach is completely independent of stochastic approximation technique and offers a new solution direction.

The rest of the paper is organized as follows. Section II states the system model. Section III presents the proposed algorithm. 
Section IV shows the numerical studies. Finally, some concluding remarks are given in Section V.

\section{SYSTEM MODEL}

We consider here a mobile cellular communication system with $M$ active users. The $i$-th user homes on to a base station (BS), labeled as $c_{i}$. For different $i$ and $j, c_{i}$, and $c_{j}$ could be identical or different, depending on the multiple access method used. For each user, there is a pair of orthogonal channels for mobile-to-base (uplink) and base-to-mobile (downlink) communication. Since there is no interference between the uplink and downlink channels, we consider power control only for the uplink channels in this paper. The results can be applied to the case for downlink channels as well.

Propagation loss and multipath effects are captured by the link gains. The notation $G_{i j}(t)$ is used to represent the path gain from the transmitter of the $j$-th user to the BS $c_{i}$. The $M$ by $M$ matrix, $G(t)=\left\{G_{i j}(t)\right\}$, is known as uplink gain matrix. In wireless communication, the link quality is usually measured by the SIR. Under our model, the SIR of user $i$ at time $t, \Gamma_{i}(t)$, is given by

$$
\Gamma_{i}(t)=\frac{G_{i i}(t) P_{i}}{\sum_{j \neq i} G_{i j}(t) P_{j}+\eta_{i}^{0}}
$$

where $\eta_{i}^{0}$ is the receiver noise at BS $i$ and $P_{i}$ represents the transmit power level of the $i$-th user. For simplicity of discussion, denote

$$
Z_{i j}(t) \triangleq \frac{G_{i j}(t)}{G_{i i}(t)} .
$$

Then (1) can be rewritten as

$$
\Gamma_{i}(t)=\frac{P_{i}}{\sum_{j \neq i} Z_{i j}(t) P_{j}+\eta_{i}(t)}
$$

where $\eta_{i}(t)=\eta_{i}^{0} / G_{i i}(t)$. The following assumption is made throughout the paper.

Assumption 2.1: The gain $Z_{i j}(t)$ is a stochastic, time-varying process with lower bound $\underline{Z}_{i j}$ and upper bound $\bar{Z}_{i j}$, respectively, i.e.,

$$
\underline{Z}_{i j} \leq Z_{i j}(t) \leq \bar{Z}_{i j}
$$

Similarly, the receiver thermal noise $\eta_{i}(t)$ has a lower bound $\underline{\eta}_{i}$ and an upper bound $\bar{\eta}_{i}$, respectively, i.e.,

$$
0 \leq \underline{\eta}_{i} \leq \eta_{i}(t) \leq \bar{\eta}_{i}
$$

In view of (4) and (1), it follows that

$$
\begin{aligned}
\frac{P_{i}}{\sum_{j \neq i} \bar{Z}_{i j} P_{j}+\bar{\eta}_{i}} & \leq \frac{P_{i}}{\sum_{j \neq i} Z_{i j}(t) P_{j}+\eta_{i}(t)} \\
& \leq \frac{P_{i}}{\sum_{j \neq i} \underline{Z}_{i j} P_{j}+\underline{\eta}_{i}} .
\end{aligned}
$$

We denote

$$
\bar{\Gamma}_{i} \triangleq \frac{P_{i}}{\sum_{j \neq i} \bar{Z}_{i j} P_{j}+\bar{\eta}_{i}}
$$

where $\bar{\Gamma}_{i}$ is known as the lower bound SIR of $\Gamma_{i}(t)$.
In addition, we define

$$
\begin{aligned}
& \alpha_{i j} \triangleq \frac{Z}{i j}_{\bar{Z}_{i j}}, \\
& \alpha_{i} \triangleq \min _{j}\left\{\alpha_{i j}\right\}
\end{aligned}
$$

where $\underline{Z}_{i j}$ and $\bar{Z}_{i j}$ are the minimum and maximum values of $Z_{i j}(t)$ respectively. It is assumed that the time varying channel has a system bound. Consequently, the upper and lower bounds on $Z_{i j}(t)$ can be determined in accordance with, for example, channel statistics, or evaluated from environment parameters. Following (8) and (9), $\alpha_{i} \leq \alpha_{i j} \leq 1$. One can interpret $\alpha_{i}$ as an indicator of the amplitude of link gain fluctuations. It represents how much the time varying gains will change. More specifically, the larger the $\alpha_{i}$ is, the less the link gains vary. The smaller the $\alpha_{i}$ is, the more the link gains change.

In practice, it is impossible to measure the $\operatorname{SIR}, \Gamma_{i}(t)$, exactly, since measurement errors cannot be completely avoided. This should be included in the system model. We assume that the measured or estimated SIR, $y_{i}(t)$, contains an additive noise and is related to $\Gamma_{i}(t)$ via the equation

$$
y_{i}(t)=\Gamma_{i}(t)+v_{i}(t)
$$

where $v_{i}(t)$ is a random noise process. The only assumption on the noise process is that it has a known lower bound, $\underline{v}_{i}$, and a known upper bound, $\bar{v}_{i}$. That is,

$$
\underline{v}_{i} \leq v_{i}(t) \leq \bar{v}_{i} .
$$

In this paper, the channel link gain is modeled as a product of a distance dependent path loss and a shadow fading component for numerical studies. Multipath fading is assumed to be resolved by appropriate coding and interleaving techniques [16], [20]. As a result, $G_{i j}(t)$ varies in accordance with shadow fading only, and the effect of multipath fading is averaged out. For each time instant, we have

$$
G_{i j}(t)=\frac{10^{-A_{i j}(t) / 10}}{d_{i j}^{\alpha}(t)}
$$

where $d_{i j}(t)$ is the distance between BS $i$ and its user $j$, and $\alpha$ is the path loss exponent. According to Gudmundson [20], $A_{i j}(t)$ can be modeled by the following Gauss-Markov process [21]

$$
A_{i j}(t+1)=\rho A_{i j}(t)+\sqrt{1-\rho^{2}} W(t)
$$

where $\rho$ is the correlation coefficient in the fading and $W(t)$ has a normal distribution with mean zero and variance $\sigma^{2}$. This model is employed for the performance evaluation.

Remark 2.1: It is worth pointing out that the proposed algorithm is not limited to the above channel model, but can operate under different time varying systems as long as the power control problem can be defined by the system bounds.

Remark 2.2: For systems without explicit upper and lower bounds of link gains, one of the solutions is to set $\alpha_{i}$ and the target region according to the distribution of link gains. For example, with the Gauss-Markov model, based on (12) and (13), we can determine the thresholds from an estimated $\alpha_{i}$ in a 
preferable confidence interval. This approach is adopted in our numerical studies for a practical consideration.

\section{POWER CONTROL FOR TIME VARYING Systems}

Before stating the proposed algorithm, it is helpful to recall some background results from the work [12]. In the following discussions, the time index $t$ is restricted to an iteration approach, i.e., $t=1,2, \ldots$, for a discrete time model.

\section{A. Algorithm for Fixed Link Gains}

In [12], the SIR is assumed to be known exactly and the link gains are not time varying. Each mobile unit adjusts its transmit power $P_{i}^{(n+1)}$ at iteration $(n+1)$ in a discrete time manner according to the following updating rule

$$
P_{i}^{(n+1)}= \begin{cases}\delta P_{i}^{(n)}, & \text { if } \Gamma_{i}^{(n)}<\delta^{-1} \gamma_{i} \\ \delta^{-1} P_{i}^{(n)}, & \text { if } \Gamma_{i}^{(n)}>\delta \gamma_{i} \\ P_{i}^{(n)}, & \text { otherwise }\end{cases}
$$

where $\delta>1$. It can be seen from the above description that a SIR target region $\left[\delta^{-1} \gamma_{i}, \delta \gamma_{i}\right]$ is defined for each mobile unit. If the SIR is below the region, the BS will instruct the mobile unit to raise the power to the next higher level. If the SIR is above the region, the power will be adjusted downwards by one level. The upper threshold is $2 \delta^{(\mathrm{dB})}$ higher than the lower threshold. (The sign $x^{(\mathrm{dB})}$ is used to denote the decibel value of $x$, i.e., $x^{(\mathrm{dB})}=10 \log _{10} x$.)

It has been proved in [12] that under suitable conditions, the algorithm given by (14) converges to a fixed point $P_{0}$, which is the optimal solution for power control. In the following subsection we are concerned with the power control for the system with time varying link gains.

\section{B. Algorithm for Time Varying Systems}

For time varying link gains with SIR measurement errors, we modify (14) to define an updating algorithm as follows. The transmit power of user $i$ at each iteration $n$ is adjusted in a discrete time manner according to

$$
P_{i}^{(n+1)}= \begin{cases}\delta P_{i}^{(n)}, & \text { if } y_{i}^{(n)}<\delta^{-1} \gamma_{i}+\underline{v}_{i} \\ \delta^{-1} P_{i}^{(n)}, & \text { if } y_{i}^{(n)}>\delta \gamma_{i}^{0}+\bar{v}_{i} \\ P_{i}^{(n)}, & \text { otherwise }\end{cases}
$$

where $\delta>1, \gamma_{i}^{0}=\alpha_{i}^{-1} \gamma_{i}$ and $\alpha_{i}$ is defined as in (9). This algorithm is a generalized version of the previous work (14), which is a special case of (15) when $\alpha_{i}^{-1}$ is equal to 1 . Since the algorithm (14) is only applicable to time invariant systems, we have extended the result to handle time varying systems. Besides, measurement error is addressed for practical consideration. As a result, the SIR thresholds include the lower and upper bounds of the measurement noises.

The designated target region is associated with the variation of system link gains. The width of target region $\left[\delta^{-1} \gamma_{i}+\underline{v}_{i}, \delta \gamma_{i}^{0}+\right.$ $\left.\bar{v}_{i}\right]$ increases as $\alpha_{i}^{-1}$ since $\gamma_{i}^{0}=\alpha_{i}^{-1} \gamma_{i}$. A small $\alpha_{i}$ implies that the link gain $G_{i j}$, as well as $Z_{i j}$, will vary in a large range.
Consequently, we need to set the target region wide enough. Otherwise, it may lead to an uncertainty in the SIR convergence.

Remark 3.1: Since $\alpha_{i}^{-1} \geq 1$, the presented algorithm (15) for time varying systems generally has a wider SIR target region than that in (14) for a time invariant system.

Remark 3.2: It is worth emphasizing that the main objective of the proposed algorithm is to enable all users to be above the minimum performance threshold.

1) Quantization of Power Level: The following basic assumption is made throughout the paper.

Assumption 3.1: For any $i$, there exists a nonnegative vector $P^{*}$ such that

$$
\bar{\Gamma}_{i}\left(P^{*}\right) \equiv \frac{P_{i}^{*}}{\sum_{j \neq i} \bar{Z}_{i j} P_{j}^{*}+\bar{\eta}_{i}}=\gamma_{i}
$$

for $i=1,2, \ldots, N$.

We say the set of SIR targets $\gamma_{i}$, where $i=1, \ldots, N$, are lower bound feasible if there is a nonnegative finite vector $P^{*}$ satisfies (16). As in [12], the power level in our model is quantized. The difference between two consecutive levels is equal to $\delta^{(\mathrm{dB})}$. Since the power has been quantized into discrete levels, we can only require the SIR to converge to a specified target region, instead of an exact target value. In the following, we will establish a convergence result for the proposed algorithm. Our approach is based on the idea presented in [12]. However, as the gains $Z_{i j}$ are time varying and SIR estimates are corrupted, the proof is more complicated.

Lemma 3.1: If there exists a power vector $P^{*}$ such that (16) holds for all $i$, then there exists a quantized power vector $\hat{P}$ such that

$$
\delta^{-1} \gamma_{i} \leq \Gamma_{i}(n, \hat{P}) \leq \delta \gamma_{i}^{0}
$$

for all $i$, where $\gamma_{i}^{0}=\alpha_{i}^{-1} \gamma_{i}$.

Proof: Given $P_{i}^{*}$, we can always find one and only one discrete power level $\hat{P}_{i}$ such that $\delta^{-1 / 2} P_{i}^{*} \leq \hat{P}_{i}<\delta^{1 / 2} P_{i}^{*}$. Let $\hat{P}$ be the quantized vector corresponding to $P^{*}$. Then,

$$
\begin{aligned}
\Gamma_{i}(n, \hat{P}) & \equiv \frac{\hat{P}_{i}}{\sum_{j \neq i} Z_{i j}(n) \hat{P}_{j}+\eta_{i}(n)} \\
& \geq \frac{\hat{P}_{i}}{\sum_{j \neq i} \bar{Z}_{i j} \hat{P}_{j}+\bar{\eta}_{i}} \\
& \geq \frac{P_{i}^{*} \delta^{-1 / 2}}{\sum_{j \neq i} \bar{Z}_{i j} P_{j}^{*} \delta^{1 / 2}+\bar{\eta}_{i}} \\
& \geq \delta^{-1} \frac{P_{i}^{*}}{\sum_{j \neq i} \bar{Z}_{i j} P_{j}^{*}+\bar{\eta}_{i}} \\
& =\delta^{-1} \gamma_{i} .
\end{aligned}
$$

On the other hand,

$$
\begin{aligned}
\Gamma_{i}(n, \hat{P}) & \leq \frac{\hat{P}_{i}}{\sum_{j \neq i} \underline{Z}_{i j} \hat{P}_{j}+\underline{\eta}_{i}} \\
& \leq \frac{P_{i}^{*} \delta^{1 / 2}}{\sum_{j \neq i} \underline{Z}_{i j} P_{j}^{*} \delta^{-1 / 2}+\underline{\eta}_{i}} \\
& \leq \delta \frac{P_{i}^{*}}{\sum_{j \neq i} \underline{Z}_{i j} P_{j}^{*}+\underline{\eta}_{i}} .
\end{aligned}
$$


From (8), $\underline{Z}_{i j}=\bar{Z}_{i j} \alpha_{i j} \geq \bar{Z}_{i j} \alpha_{i}$ and $\underline{\eta}_{i} \geq \bar{\eta}_{i} \alpha_{i}$. Thus,

$$
\begin{aligned}
\Gamma_{i}(n, \hat{P}) & \leq \delta \frac{P_{i}^{*}}{\sum_{j \neq i} \alpha_{i j} \bar{Z}_{i j} P_{j}^{*}+\underline{\eta}_{i}} \\
& \leq \delta \frac{P_{i}^{*}}{\sum_{j \neq i} \alpha_{i} \bar{Z}_{i j} P_{j}^{*}+\alpha_{i} \underline{\eta}_{i}} \\
& =\delta \alpha_{i}^{-1} \frac{P_{i}^{*}}{\sum_{j \neq i} \bar{Z}_{i j} P_{j}^{*}+\bar{\eta}_{i}} \\
& =\delta \gamma_{i}^{0} .
\end{aligned}
$$

The Proof of Lemma 3.1 is now established.

One can see from the above Lemma that a feasible solution exists if for all users $i$ the target region is $\left[\delta^{-1} \gamma_{i}, \delta \gamma_{i}^{0}\right]$.

2) Convergence Property: In this section we study the convergence property of the proposed algorithm (15). For the convenience of discussion, we make the following definitions,

$$
\begin{aligned}
\Gamma_{i}^{(n)} & \equiv \frac{P_{i}^{(n)}}{\sum_{j \neq i} Z_{i j}^{(n)} P_{j}^{(n)}+\eta_{i}^{(n)}} \\
\Gamma_{i}^{(n)}(\hat{P}) & \equiv \frac{\hat{P}_{i}}{\sum_{j \neq i} Z_{i j}^{(n)} \hat{P}_{j}+\eta_{i}^{(n)}}
\end{aligned}
$$

where $Z_{i j}^{(n)}$ and $\eta_{i}^{(n)}$ are the values of $Z_{i j}(t)$ and $\eta_{i}(t)$ at the $n$-th iteration in the discrete time manner.

Theorem 3.1: If there exists a power vector $P^{*}$ such that (16) holds for all $i$, then under the fixed step algorithm (15), the power vector, $P^{(n)}$, at any iteration $n$ has an upper bound and a lower bound which depend only on the gain matrix and the initial power vector.

Proof: The proof follows from a straightforward modification of the proof in [12].

Lemma 3.2: Let $y_{j}^{(r)}$ be the value of $y_{j}(t)$ at $r$-th iteration. If $P_{j}^{(m)} \geq \delta^{x} P_{j}^{(n)}$ and $y_{j}^{(r)}<\delta^{-1} \gamma_{j}+\underline{v}_{j}$, for $r<m<n$ and $x \geq 1$, then there exists $k \neq j$ such that $P_{k}^{(s)} \geq \delta^{x+1} P_{k}^{(l)}$, where $r \leq s<m \leq l<n$.

Proof: From the lemma assumption, it follows that $\Gamma_{j}^{(r)}<$ $\delta^{-1} \gamma_{j}$ where

$$
\Gamma_{j}^{(r)}=\frac{P_{j}^{(r)}}{\sum_{i \neq j} Z_{j i}^{(r)} P_{i}^{(r)}+\eta_{j}^{(r)}} .
$$

If $P_{j}^{(r)}<\delta^{-1} P_{j}^{(m)}$, then there exists $s$ such that $r<s<m, P_{j}^{(s)}=\delta^{-1} P_{j}^{(m)}$, and $y_{j}^{(s)}<\delta^{-1} \gamma_{j}+\underline{v}_{j}$. Thus, $\Gamma_{j}^{(s)}<\delta^{-1} \gamma_{j}$. If $P_{j}^{(r)} \geq \delta^{-1} P_{j}^{(m)}$, we let $s=r$.

Since $P_{j}^{(m)} \geq \delta^{x} P_{j}^{(n)}$, there exists $l$, where $m \leq l<n$, such that $P_{j}^{(l)}=\delta P_{j}^{(n)}$ and $y_{j}^{(l)}>\delta \gamma_{j}^{0}+\bar{v}_{j}$. Thus, $\Gamma_{j}^{(l)}>\delta \gamma_{j}^{0}$. Therefore,

$$
P_{j}^{(s)} \geq \delta^{-1} P_{j}^{(m)} \geq \delta^{x-1} P_{j}^{(n)}=\delta^{x-2} P_{j}^{(l)} .
$$
i.e.,

Denote the interference at the $n$-th iteration at BS $j$ by $I_{j}^{(n)}$,

$$
I_{j}^{(n)} \equiv \sum_{k \neq j} Z_{j k}^{(n)} P_{k}^{(n)}+\eta_{j}^{(n)} .
$$

Since $y_{j}^{(s)}<\delta^{-1} \gamma_{j}+\underline{v}_{j}$ (thus, $\Gamma_{j}^{(s)}<\delta^{-1} \gamma_{j}$ ) and $y_{j}^{(l)}>$ $\delta \gamma_{j}^{0}+\bar{v}_{j}$ (thus, $\Gamma_{j}^{(l)}>\delta \gamma_{j}^{0}$ ), by (24), we have

$$
\delta \gamma_{j}^{0}<\frac{P_{j}^{(l)}}{I_{j}^{(l)}} \leq \frac{P_{j}^{(s)}}{I_{j}^{(l)} \delta^{x-2}}<\frac{I_{j}^{(s)} \gamma_{j} \delta^{-1}}{I_{j}^{(l)} \delta^{x-2}}
$$

where $I_{j}^{(l)}$ is the value of $I_{j}(t)$ at $l$-th iteration. It follows that

$$
\delta^{x} I_{j}^{(l)}<\alpha_{j} I_{j}^{(s)} \leq \alpha_{j} I_{j}^{(s)} \leq \alpha_{j} \bar{I}_{j}^{(s)} \leq \underline{I}_{j}^{(s)}
$$

where $\quad \bar{I}_{j}^{(s)}=\sum_{k \neq j} \bar{Z}_{j k} P_{k}^{(s)}+\bar{\eta}_{j} \quad$ and $\quad \underline{I}_{j}^{(s)}=$ $\sum_{k \neq j} \underline{Z}_{j k} P_{k}^{(s)}+\underline{\eta}_{j}$. On the other hand, note that

$$
I_{j}^{(l)} \geq \sum_{k \neq j} \underline{Z}_{j k} P_{k}^{(l)}+\underline{\eta}_{j} \equiv \underline{I}_{j}^{(l)}
$$

From (27) and (28), we obtain

$$
\delta^{x}\left(\sum_{k \neq j} \underline{Z}_{j k} P_{k}^{(l)}+\underline{\eta}_{j}\right)<\sum_{k \neq j} \underline{Z}_{j k} P_{k}^{(s)}+\underline{\eta}_{j} .
$$

Which implies that there exists $k$ such that

$$
P_{k}^{(s)}>\delta^{x} P_{k}^{(l)} .
$$

Since the power level is quantized into discrete levels with step $\delta$, we have

$$
P_{k}^{(s)} \geq \delta^{x+1} P_{k}^{(l)} .
$$

This completes the proof.

Theorem 3.2: Consider a time varying system defined by (1)-(5). Suppose Assumption 2.1 and 3.1 hold, then the fixed step power control algorithm given by (15) converges to a fixed point $\hat{P}$ such that (17) is satisfied.

Proof: Since the power level is quantized into fixed step size, by Theorem 3.2, the set of reachable states is finite. We denote it by $S=\left\{S_{1}, \ldots, S_{N_{0}}\right\}$. Let $S^{*}=\left\{S_{1}, \ldots, S_{N_{1}}\right\}$ be the subset that consists of states which appear infinitely often. There exists $n_{0}$ such that, for all $n>n_{0}$, only elements in $S^{*}$ can appear. If the algorithm does not converge, $N_{1}>1$. This implies that there exists $S_{1} \neq S_{2}$, where $S_{1}, S_{2} \in S^{*}$. Let $N_{i} \gg N_{0}$. For each $S_{i}$, there exists a time sequence $T_{1}^{i}(n)<\cdots<T_{N_{i}}^{i}(n)$ such that $P^{\left(T_{1}^{i}(n)\right)}=\cdots=P^{\left(T_{N_{i}}^{i}(n)\right)}$

Define an iteration interval as a basic block in which every $S_{i}$ appears at least twice and, between the first and last appearance of a state $S_{i}$, all the other states $S_{j}$, where $i \neq j$, must appear at least once. Secondly, as $P^{(n)}$ is nonzero and bounded, there exists a positive integer $K$ such that $\delta^{K}=$ $\max _{S_{i}, S_{j} \in S, k}\left\{S_{i}(k) / S_{j}(k)\right\}<\infty$, where $S_{i}(k)$ denotes the state $i$ of user $k$, because $S_{i} \neq 0$ and $S$ is finite.

Let $B_{1}$ be a basic block that starts at $n=n_{0}$. Construct a sequence of $K$ consecutive basic blocks $\left\{B_{1}, B_{2}, \ldots, B_{K}\right\}$. 
TABLE I

Threshold on $\alpha_{i}$ IN Decibel Value for Different Outage Probabilities

\begin{tabular}{c|c|c|c|c|c|c}
\hline Outage Probability & $\rho=0.94, n=100$ & $\rho=0.51, n=100$ & Rayleigh, $n=100$ & $\rho=0.94, n=300$ & $\rho=0.51, n=300$ & Rayleigh, $n=300$ \\
\hline 0.0001 & -31.8256 & -33.5436 & -39.9817 & -34.1834 & -37.2264 & -43.5651 \\
\hline 0.001 & -28.7850 & -31.7666 & -33.9937 & -32.3577 & -34.0777 & -37.1704 \\
\hline 0.01 & -26.2236 & -29.3964 & -29.0096 & -29.5115 & -31.8085 & -32.1659 \\
\hline 0.1 & -22.9473 & -26.7478 & -23.9535 & -26.7111 & -29.2706 & -26.9312 \\
\hline 0.2 & -21.7762 & -25.7808 & -22.3093 & -25.6542 & -28.3644 & -25.2775 \\
\hline 0.3 & -21.0000 & -25.1519 & -21.3188 & -24.9471 & -27.7771 & -24.2472 \\
\hline 0.4 & -20.4012 & -24.6427 & -20.5313 & -24.3825 & -27.3082 & -23.4715 \\
\hline 0.5 & -19.8656 & -24.1941 & -19.8550 & -23.8891 & -26.8934 & -22.8184 \\
\hline 0.6 & -19.3522 & -23.7702 & -19.2615 & -23.4374 & -26.5080 & -22.2134 \\
\hline 0.7 & -18.8475 & -23.3520 & -18.6563 & -22.9714 & -26.1219 & -21.6252 \\
\hline 0.8 & -18.2659 & -22.8972 & -18.0300 & -22.4553 & -25.7020 & -21.0055 \\
\hline 0.9 & -17.5415 & -22.2901 & -17.2541 & -21.7854 & -25.1581 & -20.2076 \\
\hline
\end{tabular}

Look at $B_{K}$, there exists states $S_{1}, S_{2}$ and time instances in $B_{K}$ such that $P^{\left(n_{1}\right)}=S_{1}=P^{\left(n_{1}-T_{1}\right)}$ and $P^{\left(m_{1}\right)}=S_{2}$, where $n_{1}-$ $T_{1}<m_{1}<n_{1}$. Moreover, we can assume $P_{i}^{\left(m_{1}\right)}=\delta P_{i}^{\left(n_{1}\right)}$ for some $i$. (If $P_{i}^{\left(m_{1}\right)}=\delta^{-1} P_{i}^{\left(n_{1}\right)}$, we can reverse the roles of $S_{1}$ and $S_{2}$.) Thus, there exists $r$ such that $y_{i}^{(r)}<\delta^{-1} \gamma_{i}+\underline{v}_{i}$ (thus, $\Gamma_{i}^{(r)}<\delta^{-1} \gamma_{i}$ ), where $n_{1}-T_{1} \leq r<m_{1}$. By Lemma 3.2, there exists a user $j(j \neq i)$ such that $P_{j}^{\left(s_{1}\right)}=\delta^{2} P_{j}^{\left(t_{1}\right)}$, where $r \leq$ $s_{1}<m_{1} \leq t_{1}<n_{1}$.

In $B_{K-1}$, one can find time instances $m_{2}$ and $n_{2}$ such that $P^{\left(m_{2}\right)}$ and $P^{\left(n_{2}\right)}$ are in the same states as $P^{\left(s_{1}\right)}$ and $P^{\left(t_{1}\right)}$ respectively. Thus, we have $P_{j}^{\left(m_{2}\right)}=\delta^{2} P_{j}^{\left(n_{2}\right)}$. By repeating the argument, there exists a user $k$ such that $P_{k}^{\left(s_{2}\right)}=\delta^{3} P_{k}^{\left(t_{2}\right)}$, where $s_{2}<m_{2} \leq t_{2}<n_{2}$. Iteratively, one can find a user $l$ in $B_{1}$ such that $P_{l}^{\left(s_{K}\right)}=\delta^{K+1} P_{l}^{\left(t_{K}\right)}$, where $n_{0} \leq s_{K}<t_{K}$. This leads to a contradiction, since for any $P_{i}^{(m)}=\delta^{x} P_{i}^{(n)}, x$ is upper bounded by $K$. The contradiction implies that the fixed step algorithm converges. Thus, the theorem is established.

\section{NUMERICAL STUDIES}

The underlying system is assumed to have a standard hexagonal cellular layout with 16 cochannel cells [6] corresponding to a reuse pattern of 7 . Within each cell, there is a mobile station (MS) communicating with its nearest BS. The location of the MS is distributed uniformly inside the cell. We only consider the dynamics of shadow fading and assume the distance between the $i$-th BS and the $j$-th MS is a constant. The standard deviation $\sigma$ in shadow fading is 4 . The path loss exponent is 4 . The correlation coefficient $\rho$ in the fading model (13) is to characterize the link gain evolution due to the mobility and speeds of transmitters or receivers. For example, in a GSM system, $\rho$ of 0.51 and 0.94 refer to a MS traveling in an urban area with a speed of 100 and $10 \mathrm{~km} / \mathrm{h}$, respectively [17]. We are interested in the region $0.51<\rho<1$.

For acceptable link quality, we assume the threshold $\gamma_{i}$ is $16 \mathrm{~dB}$ for all $i$ while the receiver thermal noise is $10^{-12} \mathrm{~W}$. Each MS generates an initial power uniformly between 0.0001 and $1 \mathrm{~W}$. All the SIR values plotted in our numerical analysis are computed based on the current adjusted transmit power level attenuated with respect to the gain matrix in the next it- eration instant. This provides a stricter setup for measuring the algorithm performance than assuming the SIRs are measured instantaneously. In the proposed algorithm, we use a step size of $1 \mathrm{~dB}$.

The lower and upper thresholds of the target window are set according to $\left[\delta^{-1} \gamma_{i}+\underline{v}_{i}, \delta \gamma_{i} \alpha_{i}^{-1}+\bar{v}_{i}\right]$ as defined in (15). Samples of SIR measurement error $v_{i}$ are independently drawn from a uniform distribution such that $v_{i} \in(-1.26,1.26)$. To determine the value of $\alpha_{i}$ for the proposed algorithm with respect to the previously mentioned Gauss-Markov model, one solution is to choose it based on the distribution of $\alpha_{i}$ associated with the correlation coefficient $\rho$ and the total number of iterations $n$ considered. Table I gives the corresponding numerical values obtained for $\rho=0.94$ and 0.51 , respectively. Data for Rayleigh fading are also shown. Results are obtained after 32000 runs for 100 and 300 iterations, respectively.

The simulation model does not require an absolute bound. Instead, we set the thresholds according to an outage probability of 0.01 , i.e., $\operatorname{Pr}\left\{\alpha_{i}>\widetilde{\alpha_{i}}\right\}=0.01$, where $\widetilde{\alpha_{i}}$ denotes the chosen bound value on $\alpha_{i}$ for the algorithm. For practical consideration, a common $\widetilde{\alpha_{i}}$ is employed for all users in order to make the system manageable. However, the proposed algorithm allows distinct settings on $\alpha_{i}$ for each user.

To investigate the performance, the proposed algorithm is compared to Foschini-Miljanic algorithm [8] as given below.

$$
P_{i}^{(n+1)}=\frac{\gamma_{i} P_{i}^{(n)}}{\Gamma_{i}^{(n)}} .
$$

Fig. 1 shows a comparison under $\rho=0.94$. The convergence of the proposed algorithm to the target window is observed as long as there exists a feasible solution for the system. Comparatively, although the Foschini-Miljanic algorithm is able to adjust the power faster than the proposed one (since it uses a continuous power level) and assume prefect precision in each feedback, it cannot guarantee a convergence in the target region. This can be observed from Fig. 1, in which the minimum SIR in the Foschini-Miljanic algorithm frequently drops below the lower threshold. This could lead to frequent call drops. Comparatively, the proposed algorithm is more stable in the sense that 


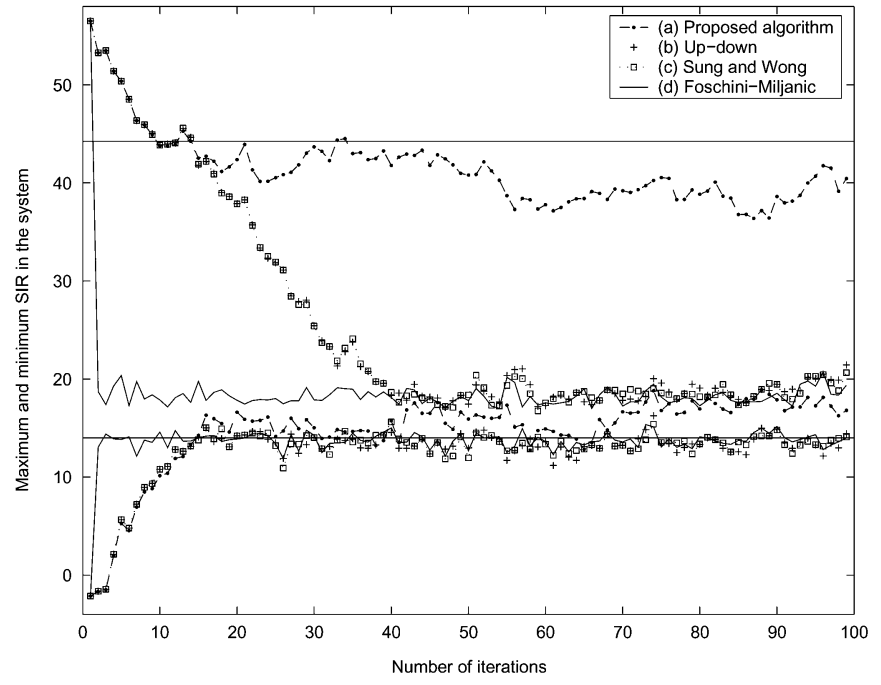

Fig. 1. Evolution of maximum and minimum SIR (decibels) with step size $\delta=1 \mathrm{~dB}$ and $\rho=0.94$. The target window is also shown.

it guarantees all the SIRs are within the expected target window and, more importantly, above the lower threshold.

At the same time, we have compared the proposed algorithm with the conventional up-down power control algorithm [22] and Sung and Wong algorithm [12]. In the up-down algorithm, transmit power is increased or decreased by a fixed amount when the SIR is below or above threshold, respectively. As shown in Fig. 1, both the up-down and Sung-Wong algorithms perform similarly to Foschini-Miljanic algorithm, but with a slower power adjustment. They do not offer the SIR performance guarantee as well. A detailed analysis of the up-down algorithm under fading can be found in [22]. Results show that it is effective only when under a slow fading.

To further demonstrate the significant difference between the proposed algorithms and the other three algorithms, we decrease $\rho$ from 0.94 to 0.51 . In this case, the link gain matrix will vary with a lower correlation in each iteration such that the channel gain fluctuation will be higher. Fig. 2 shows a typical result from which we can see the effectiveness of the proposed algorithm even under such a fluctuation. All the SIRs are still within the guaranteed target window and above the lower threshold. However, all the other three algorithms cannot provide such a QoS guarantee. Comparing Fig. 2 with Fig. 1, we can find that the SIR outage of these three algorithms is much more serious due to the higher channel fluctuation. A simulation study of the algorithms under Rayleigh fading [23] is also conducted. The result is shown in Fig. 3. The proposed algorithm is effective and outperforms the other three. As observed from Figs. 1-3, as the modified upper threshold of target window for the proposed algorithm is now moved upward, there could be an increase in the power consumption. An evaluation on this is conducted and can be found later.

Next, we investigate the effect of estimation error on the proposed algorithm. Stability of the algorithm under measurement noise is our key concern. To focus on the effect of estimation error, we set $\rho$ to 0.94 such that the weighting on the fluctuation

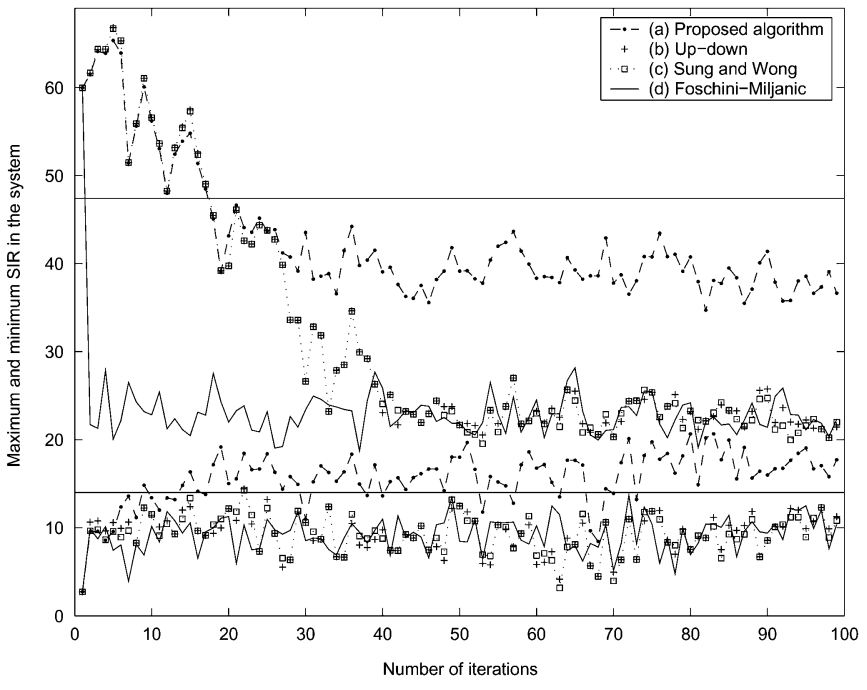

Fig. 2. Evolution of maximum and minimum SIR (decibels) with $\delta=1 \mathrm{~dB}$. The correlation coefficient $\rho$ is equal to 0.51 .

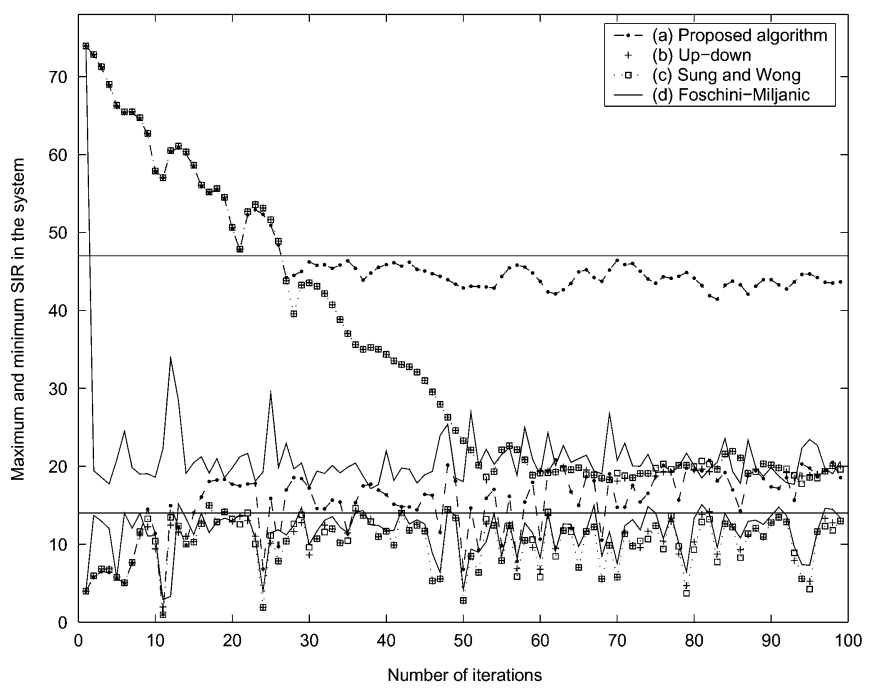

Fig. 3. Evolution of maximum and minimum SIR (decibels) with $\delta=1 \mathrm{~dB}$ under Rayleigh fading. The output sample frequency used is $1600 \mathrm{~Hz}$ and the maximum Doppler frequency shift is $200 \mathrm{~Hz}$.

due to channel variation will be relatively small. Fig. 4 shows the SIR evolutions under different estimation errors. It should be noted that since the bounds are different, the widths of target windows are different. Only the case of (a), which is the narrowest one among the three, is shown. Results in Fig. 4 show that the proposed algorithm is robust to measurement noise. We can observe the SIR convergence in all three cases.

In the following, we study the power consumption of the proposed algorithm and compare it to the Foschini-Miljanic algorithm, which is optimal in the sense that the total transmit power is being minimized. As reported in [24], for power saving, a good strategy is to employ a low initial power level and then increase it iteratively until the SIR requirement is satisfied. In the example studied, all users start from the same initial power of $-20 \mathrm{dBW}$. As shown in Fig. 5, the average transmit power of all users after convergence under the proposed algorithm is very 


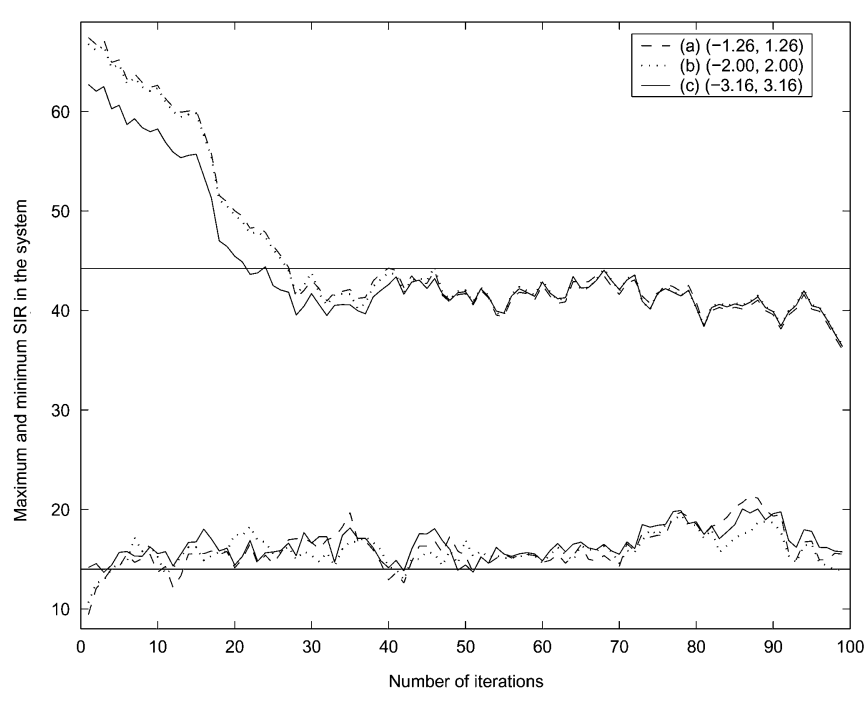

Fig. 4. Evolution of maximum and minimum SIR (decibels) under the proposed algorithm with $\delta=1 \mathrm{~dB}$ and estimation error: (a) $v_{i} \in(-1.26,1.26)$ (b) $v_{i} \in(-2.00,2.00)$. (c) $v_{i} \in(-3.16,3.16)$.

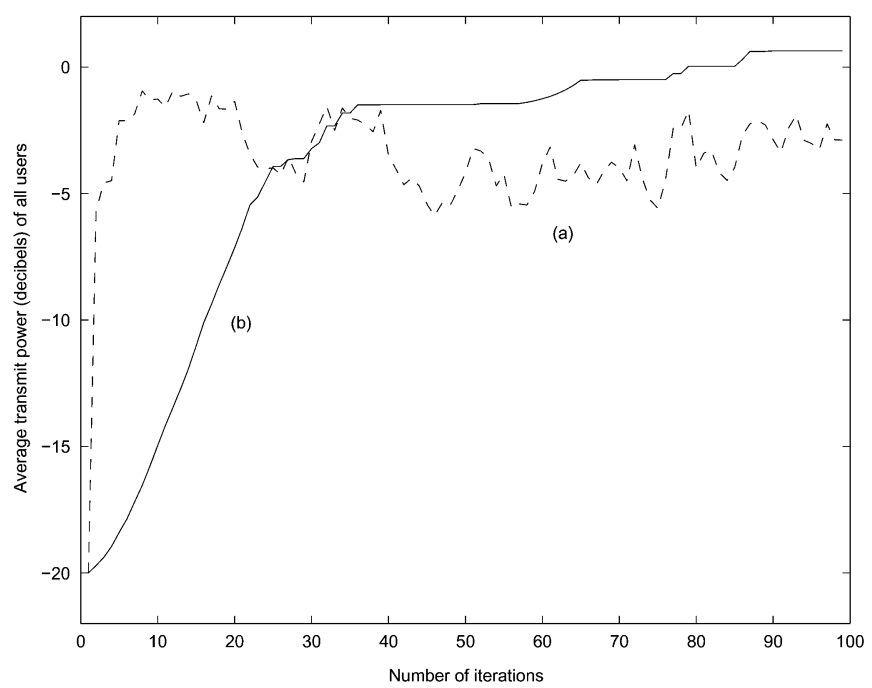

Fig. 5. The average transmit power (decibels) of all users with fixed initial power $-20 \mathrm{dBW}$ and $\delta=1 \mathrm{~dB}$. (a) Foschini-Miljanic algorithm. (b) Proposed algorithm.

close to that in the Foschini-Miljanic algorithm. This implies that the power consumption in the proposed algorithm can be quite ideal if the initial power is set low enough initially. Fig. 6 shows the evolution of maximum and minimum transmit power among all users.

Next, we will demonstrate the effectiveness of the proposed algorithm under different target threshold settings with respect to the outage probability on the specified $\alpha_{i}$. All the system parameters are common except the target thresholds. As shown in Fig. 7, the proposed algorithm can work well even when the setting is not very precise. However, it should be noted that a larger outage probability on the chosen value of $\alpha_{i}$ implies a higher chance the algorithm does not converge to the target window, as the upper threshold is too low. A loose requirement

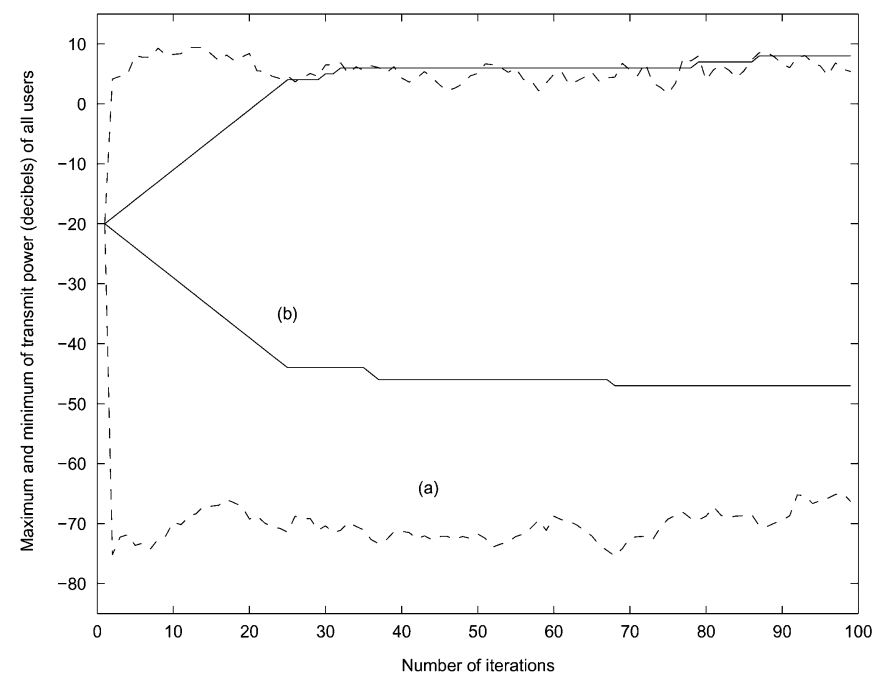

Fig. 6. Evolution of maximum and minimum transmit power (decibels) with fixed initial power $-20 \mathrm{dBW}$ and $\delta=1 \mathrm{~dB}$. (a) Foschini-Miljanic algorithm (b) Proposed algorithm.

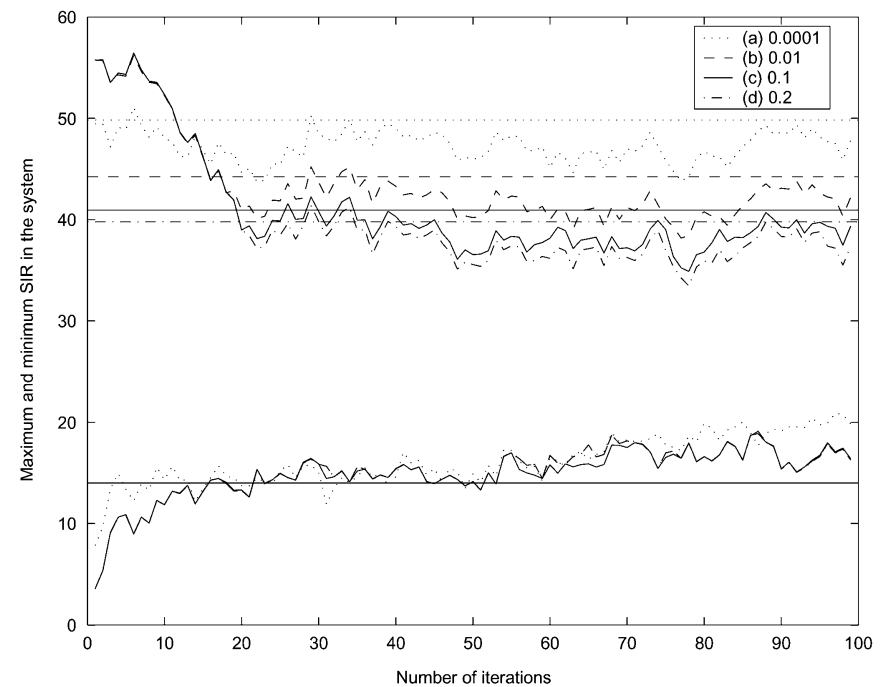

Fig. 7. Evolution of maximum and minimum SIR (decibels) with $\delta=1 \mathrm{~dB}$, $\rho=0.94, v_{i} \in(-1.26,1.26)$, and outage probabilities. (a) 0.0001 . (b) 0.01 . (c) 0.1 . (d) 0.2 . The corresponding target windows are also shown while the lower threshold is common.

on the setting may lead to system instability. However, it may also allow a narrower convergence window.

As observed from Figs. 1-3, under the Foschini-Miljanic, Sung-Wong and up-down algorithms, there is a frequent SIR dropping below the lower target threshold. To alleviate this kind of problem, the idea of adding an SIR margin to signal quality target [16] can help to reduce the SIR outage probability due to channel fluctuations. A numerical analysis is conducted for the three cases of Figs. 1-3. By inspection and verification with simulations, an SIR margin of 4,8 , and $8 \mathrm{~dB}$ should be added to the cases of Figs. 1, 2, and 3, respectively so as to achieve a roughly similar minimum SIR performance as that of the proposed algorithm. Generally, a system with high channel fluctuation needs a large SIR margin, which corresponds to an increase in 
TABLE II

AVERAGED TRANSMISSION POWER CONSUMPTION NORMALIZED BY THAT IN THE PROPOSED ALGORITHM

\begin{tabular}{c|c|c|c}
\hline $\begin{array}{c}\text { Ratio of average power } \\
\text { consumption to the proposed one }\end{array}$ & $\begin{array}{c}\text { Foschini- } \\
\text { Miljanic }\end{array}$ & $\begin{array}{c}\text { Sung- } \\
\text { Wong }\end{array}$ & $\begin{array}{c}\text { Up- } \\
\text { down }\end{array}$ \\
\hline for Fig. 1 (4dB SIR Margin) & $108.95 \%$ & $99.18 \%$ & $100.79 \%$ \\
\hline for Fig. 2 (8dB SIR Margin) & $552.03 \%$ & $271.69 \%$ & $277.98 \%$ \\
\hline for Fig. 3 (8dB SIR Margin) & $392.18 \%$ & $267.14 \%$ & $277.70 \%$ \\
\hline
\end{tabular}

transmission power. Table II gives the ratios of average power consumed by users of the Foschini-Miljanic, Sung-Wong, and up-down algorithms to that used in the proposed algorithm. Results for each case are obtained with 3000 runs. The transmission power is measured from $n=50$ to 100 only in order to investigate the power consumption after an equilibrium. In the case of Fig. 1, which has a small channel fluctuation, the average power consumptions in all the algorithms are very close. In the cases of Figs. 2 and 3, which have a high channel fluctuation, the proposed algorithm consumes much less power. Besides, it is worth pointing out that the focus and approach of our work reported here is fundamentally different to that in [16].

\section{CONCLUSION}

In this paper, we propose a new algorithm to solve a power control problem for systems with time varying link gains. It is based on the fixed step approach in [12]. We assume that the link gains change randomly in a bounded region with known upper and lower bounds and the estimates of SIR are corrupted by bounded noises. The power level of each mobile terminal is adjusted in a distributed manner. The definition of the upper threshold has to be modified accordingly. Convergence of the algorithm to the target region is studied. Numerical studies show that it is applicable to realistic systems.

\section{REFERENCES}

[1] J. M. Aein, "Power balancing in systems employing frequency reuse," COMSAT Tech. Review, vol. 3, no. 2, pp. 277-299, 1973.

[2] R. W. Nettleton and H. Alavi, "Power control for a spread spectrum cellular mobile radio system," in Proc. IEEE Vehicular Technol. Conf., 1983, pp. 242-246.

[3] J. Zander, "Performance of optimum transmitter power control in cellular radio systems," IEEE Trans. Veh. Technol., vol. 41, pp. 57-62, 1992.

[4] — , "Distributed cochannel interference control in cellular radio systems," IEEE Trans. Veh. Technol., vol. 41, no. 3, pp. 305-311, 1992.

[5] S. A. Grandhi, R. Vijayan, and D. J. Goodman, "Distributed power control in cellular radio systems," IEEE Trans. Commun., vol. 42, pp. 226-228, Feb. 1994.

[6] K. H. Lam and W. S. Wong, "Distributed power balancing in cellular systems using limited control-data flow," IEEE Trans. Veh. Technol., vol. 46, no. 1 , pp. 247-252, Feb. 1997.

[7] C. W. Sung and W. S. Wong, "The convergence of an asynchronous cooperative algorithm for distributed power control in cellular systems," IEEE Trans. Veh. Technol., vol. 48, no. 2, pp. 563-570, Mar. 1999.

[8] G. J. Foschini and Z. Miljanic, "A simple distributed autonomous power control algorithm and its convergence," IEEE Trans. Veh. Technol., vol. 42, no. 4, pp. 641-646, Nov. 1993.

[9] P. S. Kumar and J. Holtzman, "Power control for a spread spectrum system with multi-user receivers," Sixth IEEE International Symposium on Personal, Indor and Mobile Radio communications, 1995. PIMRC'95, vol. 3, p. 955 , Sep. 1995.
[10] R. D. Yates and C. Y. Huang, "Integrated power control and base station assignment," IEEE Trans. Veh. Technol., vol. 44, no. 3, pp. 638-644, 1995.

[11] S. Ulukus and R. D. Yates, "Adaptive power control with MMSE multiuser detectors," in Proc. IEEE International Conference on Communications. 1997. ICC'97, vol. 1, Jun. 1997, pp. 361-365.

[12] C. W. Sung and W. S. Wong, "A distributed fixed-step power control algorithm with quantization and active link quality protection," IEEE Trans. Veh. Technol., vol. 48, no. 2, pp. 553-562, Mar. 1999.

[13] H. Zhang, W. S. Wong, W. Ge, and P. E. Caines, "A Stochastic Approximation Approach to the Power Control Problem".

[14] Y.-W. Leung, "Power control in cellular networks subject to measurement error," in IEEE Trans. Commun., vol. 44, no. 7, Jul. 1996, pp. 772-775.

[15] S. Ulukus and R. D. Yates, "Stochastic power control for cellular radio systems," IEEE Trans. Commun., vol. 46, no. 6, pp. 784-798, Jun. 1998.

[16] M. Andersin and Z. Rosberg, "Time variant power control in cellular networks," in Proc. Seventh IEEE International Symposium on Personal, Indoor and Mobile Radion Comunications, 1996, PIMRC'96, vol. 1, Oct. 1996, pp. 193-197.

[17] C. W. Sung and W. S. Wong, "Performance of a cooperative algorithm for power control in cellular systems with a time-varying link gain matrix," Wireless Networks, vol. 6, pp. 429-439, Dec. 2000.

[18] S. Kandukuri and S. Boyd, "Optimal power control in interference-limited fading wireless channels with outage-probability specifications," IEEE. Trans. Wireless Commun., vol. 1, no. 1, pp. 46-55, Jan. 2002.

[19] S. A. Grandhi and J. Zander, "Constrained power control in cellular radio systems," in Proc. IEEE 44th Vehicular Technology Conference, vol. 2, Jun. 1994, pp. 824-828.

[20] M. Gudmundson, "Correlation model for shadow fading in mobile radio systems," Electron. Lett., vol. 27, pp. 2145-2146, Nov. 1991.

[21] H. Stark and J. W. Woods, Probability, Random Processes, and Estimation Theory for Engineers. Englewood Cliffs, NJ: Prentice-Hall, 1986.

[22] L. Song, N. B. Mandayam, and Z. Gajic, "Analysis of an up/down power control algorithm for the CDMA reverse link under fading," IEEE J. Sel. Areas Commun., vol. 19, no. 2, pp. 277-286, Feb. 2001.

[23] Rec. ITU-R M.1225, Guidelines for Evaluation of Radio Transmission Technologies for IMT-2000: International Telecommunication Union, 1997.

[24] C. W. Sung, K. K. Leung, and W. S. Wong, "A quality-based fixed-step power control algorithm with adaptive target threshold," IEEE Trans. Veh. Technol., vol. 49, no. 4, pp. 1430-1439, Jul. 2000.

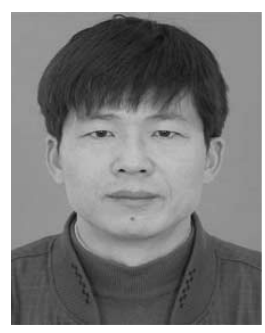

Huanshui Zhang (M'98) received the degree in mathematics from the Qufu Normal University in 1986 and received the M.Sc. and Ph.D. degrees in control theory and signal processing from the Heilongjiang University, P.R. China, and Northeastern University, P.R. China, in 1991 and 1997, respectively. He worked as a postdoctoral fellow at the Nanyang Technological University from 1998 to 2001 and Research Fellow at Hong Kong Polytechnic University from 2001 to 2003.

He joined Shandong Taishan College in 1986 as an Assistant Professor and became an Associate Professor in 1994. He joined Shandong University in 1999 as a Professor. Currently he is a Professor at Shenzhen Graduate School of Harbin Institute of Technology, HIT Campus, Shenzhen University Town, Xili, Shenzhen, P.R. China.

His research interests include optimal estimation, robust filtering and control, time delay systems, singular systems, wireless communication, and signal processing.

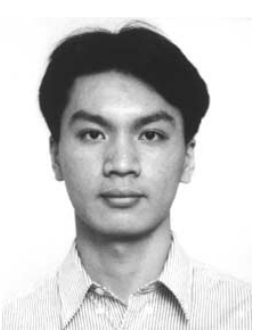

Chung Shue Chen (S'02) was born in China in 1977. $\mathrm{He}$ received the B.Eng. and M.Phil. degrees in information engineering in 1999 and 2001, respectively, from the Chinese University of Hong Kong, Shatin, Hong Kong, where he is currently working toward the Ph.D. degree.

His research interests include resource allocation, power control, traffic scheduling, QoS guarantee, and multiple access control for wireless communication systems. 


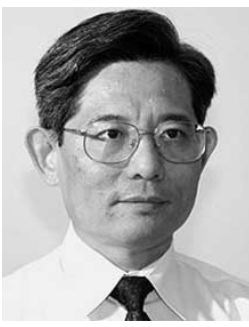

Wing Shing Wong (M'81-SM'90-F'02) received a combined Master's and Bachelor's degree (summa cum laude) from Yale University, New Haven, CT, in 1976, and the M.S. and Ph.D. degrees from Harvard University, Cambridge, MA, in 1978 and 1980 respectively.

He joined AT\&T Bell Laboratories, Holmdel, NJ, in 1982 . From 1987 to 1992 , he managed a group of technical staff working on a number of research and development projects and consulting activities. He joined the Chinese University of Hong Kong in
1992 and is now a Professor of Information Engineering. He was the Chairman of the Department of Information Engineering from 1995 to 2003. Currently, he is serving as Science Advisor at the Innovation and Technology Commission of the HKSAR government. He is also a Visiting Professor of Southeast University, Nanjing, China. He is actively involved in a variety of research and development projects including topics such as mobile communication systems, search engine development, and information issues in estimation and control. He has published over 100 refereed journal and conference papers.

Dr. Wong was an Associate Editor of the IEEE Transactions on Automatic Control for four years. He is the Co-Editor-in-Chief of a new journal, Соттиnications in Information and Systems. 